\title{
Career Counseling Chatbot on Facebook Messenger using AI
}

\author{
Nalina Suresh \\ School of Computing \\ University of Namibia \\ Windhoek, Namibia \\ nsuresh@unam.na \\ Anton Limbo \\ School of Computing \\ University of Namibia \\ Windhoek, Namibia \\ alimbo@unam.na
}

\author{
Nkandu Mukabe \\ School of Computing \\ University of Namibia \\ Windhoek, Namibia \\ ncmukabe@gmail.com
}

\author{
Aina Hauwanga \\ School of Computing \\ University of Namibia \\ Windhoek, Namibia \\ ankandi@unam.com
}

\author{
Valerianus Hashiyana \\ School of Computing \\ University of Namibia \\ Windhoek, Namibia \\ vhashiyana@unam.na
}

\begin{abstract}
Increasingly we observe that newly graduated university students struggle to find employment, often ending up in fields completely different from those that they studied in, and employees reporting low levels of satisfaction in the careers that they choose. One of the reasons for this could be a lack of adequate, useful career guidance when an individual is in university or recently graduated. Chatbots are useful resources and a topic of interest in the realm of Computer Science and AI for their ability to mimic experts in different applications, as well as being able to replicate human interaction to varying degrees.
\end{abstract}

Research suggests that the use of a chatbot in offering career counseling can serve as an efficient means to provide this useful service in environments where a dedicated career counsellor may not be available. A lack of appropriate, adequate career counseling means youth may wind up pursuing careers that are chosen for them by their parents or only chosen for the promise of high salaries. These decisions may be made without taking into consideration whether or not they align with the individual's interests and values.

This can lead to feelings of dissatisfaction in one's career, which is detrimental not only to the wellbeing of the individual but also to the overall productivity of the business in which they are hired. Hence, the development of the chatbot will better inform users, assisting them in the ways of choosing a career. This will allow them to consider careers they may not have thought available to them, that would be more fulfilling and satisfactory than one that does not align with their interests.

\section{(c) (i) $\ominus$}

This work is licensed under a Creative Commons Attribution-NoDerivs International 4.0 License.

DSMLAI '21', August 9-12, 2021, Windhoek, Namibia (C) 2020 Association for Computing Machinery. ACM ISBN 978-1-4503-8763-7/20/06...\$15.00 https:// doi.org/10.1145/3484824.3484875
This research adopted both Research methodology and Software Development methodology. Under Research Methodology, a survey was conducted to gather information about individuals' opinions regarding their possible career choices, and what types of career advice they would like to receive from the system. In addition, the latest research on career guidance was considered in forming the basis of the questions asked. This information was used in chatbot system development on the Facebook Messenger platform, using tools such as the Facebook SDK, the Messenger Platform APIs, and JavaScript, and the Wit.ai API which enables the use of Natural Language Processing, which is a use case of AI techniques.

The chatbot understands user input and give relevant and appropriate responses, reliably and in real-time. In conclusion, the results obtained are hoped to result in positive proclivity towards adopting the system, thus serving as a useful asset to any universities or institutions that might wish to utilize it.

\section{CCS CONCEPTS}

$\begin{array}{lccl}\cdot \text { Natural } & \text { Language } & \text { Processing } & \bullet \text { Artificial } \\ \text { Intelligence } & \bullet \text { Knowledge representation and reasoning }\end{array}$

\section{KEYWORDS}

Chatbot, Career Counseling, Natural language Processing.

\section{ACM Reference format:}

Nalina Suresh, Nkandu Mukabe Valerianus Hashiyana, Anton Limbo and Aina Hauwanga. 2021. Career Counseling Chabot on Facebook Messenger using AI. In Proceedings of ACM Data Science, Machine Learning and Artificial Intelligence conference (DSMLAI). Namibia, 9-12 August 2021, Jackson Kaujeua Street, Windhoek, Namibia, 10 pages. ACM Digital Library. Association for Computing Machinery, New York, NY, United States (ISBN Number: 978-1-4503-8763-7) 


\section{Introduction}

This study explores the current state of career counseling, and how a lack of adequate career counseling during tertiary education and beyond it could result in difficulty in finding employment, as well as low work satisfaction in employees. This study suggests a proposed solution of implementing career counseling chatbots, which students and graduates could use to assist them in discovering potential career paths.

A common concern of every student once they graduate is to find a career doing something they love. However, increasingly it is apparent that graduates struggle to find employment, and employees reporting low levels of satisfaction in their places of work. According to a survey done by Universum SA, $47 \%$ of young professionals were ready to move on from their current places of employment, and of the 20, 000 individuals surveyed, average job satisfaction of $6 / 10$ was reported [1].

Selecting a career that aligns with one's interests is an important factor in feeling satisfied with one's career and maintain this interest. However, most people in African homes simply choose careers chosen by their parents, which are suggested for their high salary. This lack of interest or meaningful work for an employee is a cause of job dissatisfaction.

Proper career counseling is important to help guide students to choose a suitable career they are interested in. The use of Artificial Intelligence (AI) can serve as a smart and efficient means to provide this service. AI is "the ability of a digital computer or computercontrolled robot to perform tasks commonly associated with intelligent beings" [2], such as visual perception, speech recognition, language processing, decision-making, etc. One application of this is a chatbot, which is defined as an Artificial Intelligence (AI) software that can simulate a conversation (or a chat) with a user in natural language through messaging applications, websites, and mobile apps or through the telephone [3]. They are designed to mimic human communication, allowing the user to converse with them for a variety of applications. They do this by employing Natural Language Processing, defined by [4] as a method by which computers manipulate natural language in text or speech, in order to perform the desired task. This makes them a very useful tool in a field such as career counseling. This study aims to implement a chatbot and utilize its ability to analyze patterns in user input and provide an appropriate response.

\section{$2 \quad$ Statement of the problem}

Many graduates and young employees report uncertainty in their career choices, and dissatisfaction in their current careers. It is a challenge for students to find sufficient career advice, given that campuses have a low student-to-advisor ratio, or in some cases, no dedicated advisory services at all. In most cases, students pursuing careers without useful advisory input, or merely pursuing careers due to parental influence can lead to dissatisfaction in one's career. The drawbacks of pursuing a career that does not fit one's interests - including lack of interest or personal fulfillment - affect not only the individual but also the industry in which they work. As an individual, these effects can be detrimental to their happiness and wellbeing. In terms of workplaces in general, an unhappy employee is likely to be less productive than one who feels fulfilled in their work, meaning the company itself will suffer losses. In addition, employees feeling dissatisfied in their places of work could result in a high employee turnover rate, defined as a large percentage of employees leaving a company in a given time frame. This results in lowered productivity and higher costs for the business to replace them. This research aimed to address these issues for the good of the businesses and the individuals who work there. The use of the chatbot will be beneficial as it can address the low student-toadvisor ratio, as it can handle many concurrent users. In addition, having a system with fundamental career information will allow the users to be adequately informed as they prepare to enter the work environment. Lastly, in context to the University of Namibia (UNAM), meager research studies have been conducted in this field.

\section{Objectives}

The main objective of this research was to establish a chatbot application that would provide career counseling services to students and graduates.

Further, the main objectives are associated with the supporting or sub objectives such as:

1. Identify the necessary tools required to deploy the chatbot application, including algorithms, APIs (Application Program Interfaces) and latest information on career guidance to form the basis of the questions asked.

2. To design a chatbot that is expected to understand user input and give relevant appropriate responses.

\section{Literature Review}

In the research carried out by [5], the authors aimed to create a chatbot for career guidance using AI. The system employed the use of several tools in the building of the chatbot including Dialog Flow, which is defined as a "Google API module that is used to process a natural language query and returns structured, actionable data as a result" [6] and Hidden Markov Model (HMM), which was used in Speech recognition. When processing input from the user, both systems made use of pattern matching and a process of intent classification to understand user input. Intent classification involves the "breaking down of user inputs into categories", and from those categories, the NLP algorithm put in place understands what the purpose of a certain input is.

The resulting chatbot was able to give career counseling to class 12 and similarly graduate students. However, their system was implemented as a standalone desktop application, while this chatbot system was implemented on the Facebook Messenger platform for ease of access and use. Additionally, they made use of HMM and Dialogflow to process and understand user input, while the implemented system makes use of Wit.ai's NLP capabilities to understand user input and provide a response. This was favored over [5] selection as it was easier to integrate with the chatbot system. It is also worth noting that this research paper does not 
include user evaluation of the chatbot system that was implemented. In contrast to the above system, the implemented system was tested by a sample of users to gain a better understanding of what worked well and gave room for future enhancements. In this paper by [7], the authors sought out to develop a chatbot capable of educating the user about sports and demonstrating intelligent behavior. They drew insights from different chatbot systems such as ELIZA, one of the very first chatbots created. The chatbot, which was designed in 1966 by Joseph Weizenbaum, behaved as a therapist of sorts, "rephrasing statements of the user and posing them back as questions". ELIZA helped the authors understand how reframing input could make conversations more human-like. They also drew insights from ALICE (Artificial Linguistic Internet Computer Entity) - a chatbot inspired by ELIZA - to understand how to make use of Artificial Intelligence Mark-up Language (AIML) for their system, and Siri - a virtual personal assistant developed by Apple - which helped to understand the limitations of language processing in a speech-tospeech agents.

This research paper implements the use of text-to-speech that makes use of various tools, such as the Google speech recognition API to convert spoken input to text, a spoken language understanding module and semantic representation to extrapolate the meaning of the query, and the Google text-to-speech synthesis, to return output as speech as well. However, the proposed system will opt to implement a purely text-based system for several reasons, the first being to reduce the complexity of the system. Secondly, the use of fewer APIs and frameworks allow for faster processing of the data, allowing the chatbot to deliver responses in a shorter timeframe than if it had to process speech to text and vice versa. Thirdly, the implemented chatbot can easily be accessed on a smartphone, many of which come with inbuilt speech-to-text systems and users who may wish to speak to the chatbot can use this feature on their phone. Their smartphone will be able to convert the audio to text and send it to the chatbot without the need for a dedicated speech-to-text framework to be built. The resulting chatbot implemented in this research paper was an Android OS system that took in speech input and returned relevant responses to the user in speech format as well. Though the subject matter of the system differs from the implemented career counseling chatbot, the research still provided important insight about how to build a chatbot and allowed the researcher to identify underlying frameworks that would not be necessary to implement an efficient system.

The research paper [8] proposed an intelligent career counseling chatbot that converses with the user using both text and speech data. To build the bot, artificial intelligence algorithms were used to analyse and understand user queries and provides a valid output. Their system implemented Natural Language Processing and a customised pattern-matching algorithm to generate an appropriate answer to the user query.

Similar to this study, the implemented system also made use of Natural Language Processing, and a pattern-matching approach that locates key phrases in user input to generate the output. Additionally, both systems store keywords in a knowledge base, defined in this paper as "a technology used by a computer system to store complex structured and unstructured information." In contrast, however, their system utilized both text and speech data for both the input and the output, whereas the proposed implemented system is limited to text only. This simplified the process of developing and implementing the system. In addition, it reduced possible errors that might be introduced by accents that may make speech input hard to process. The result of this research is a proposed intelligent chatbot, which gave valuable insights to the researcher on how to implement a similar system, outlining useful resources and features that could be implemented in different or more efficient ways. The choice to implement an aptitude test that would assist users in choosing a future career was in part constructed after reviewing this paper.

In this study, [9] described the design and implementation of a university counseling chatbot that provided answers to queries related to the field of Engineering. It covered a range of applications, including queries relating to admission to the university, available courses, job placement, and many more. NLP techniques were applied to the collected dataset, developed in the JSON format, and trained using the feedforward neural model. The application was deployed on the Facebook Messenger interface. Similarly, the proposed system was also deployed on the Facebook Messenger application for its popularity and availability and made use of NLP and JSON files to store responses. In addition, the proposed system will adopt the same end-user testing process. In this study, the authors conducted two-phase end-user testing in which testing was done after an initial development iteration and then conducted again after adding functionality to the system. This will be adopted in the proposed study to gauge how well the features meet the requirements. The system created in this study implemented a feed-forward neural network framework to train the model, on the contrary, the implemented system made use of pretrained NLP models available on the Wit.ai API. Their system required a data set consisting of "62 intent tags and 1059 query patterns" that needed to be stored in a JSON file, and all input queries had to be checked against this dataset to identify a matching pattern. However, the implemented system only required six intent tags to be created in the Wit.ai interface, and the query patterns were automatically processed without the need for manually creating them. Furthermore, the system created in this study covered a range of applications, but only within the field of engineering at the particular institution. The proposed system's scope will be limited only to career counseling but will cater to numerous different fields of work.

The research paper [10], focused on the development of an artifact designed to assist users in looking for jobs. The system created makes use of AIML and NLP to create a chatbot that converses with the user. Notably, this study also implemented a speech-to-text feature that recorded user's voices and converted it to text input, without requiring the user to use their keyboard. Google APIs, Java programming language and customized AIML libraries specifically for the career counseling domain were used to further advance the chatbot. This paper gave insight into how to formulate and design the system, such as using agile development methodologies for the 
software development and listing some research ethics that were adhered to when working with participant data.

Similar to this study, the implemented system will make use of NLP to process user input and determine a suitable response. The system created in this study also made use of AIML and Google APIs. On the other hand, the implemented counseling chatbot made use of the resources already available on the Facebook platform. This is because the tools found on Facebook are more powerful, as it has a larger dataset of chatbot interactions as compared to the Google API. The system in this paper was implemented as a standalone offline system, whereas the proposed system will be available online. This will allow for availability to users from all around the world to easily access the system. Lastly, this study implemented speech-to-text functionality, similar to [9] listed above. However, this API slowed down response times and on occasion would crash after overuse. For this reason, and for the sake of simplifying the development process as stated above, the counseling chatbot was implemented as text-only and reported very fast response times.

In this study, [11] describe the design and development of a chatbot that provides inquiry information about a college through a text or voice-based interface. In it, they highlight the process flow of a standard chatbot, as illustrated in the flowchart shown in Figure 1.

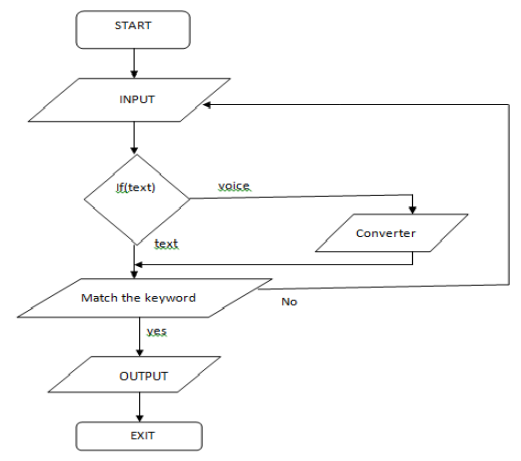

\section{Figure 1: Flow Chart Design}

This Figure 1 flow diagram was adapted and simplified by omitting the check for voice input, as the system primarily used text. This is not to say that voice cannot be used, as many smartphones come with an inbuilt voice-to-text feature which will convert a spoken message to text before sending it to the chatbot. As such, it can be omitted from the chatbot's flowchart.

They made use of the Dialogflow API for the database management and NLP functionality to understand the input given by the user. To build the interface, they made use of Unity, a "gaming platform which supports 2D as well as 3D gaming graphics" [11], which would run the application on Android devices. However, the career counseling system made use of Facebook messenger, which would be accessible on any device, and would have a more traditional chat interface, rather than a game interface. This would also allow the chatbot to be used without having to download a separate application, as it can be accessed on a browser or the Facebook app that most smartphones have preinstalled.

The research paper [12] presented the design and development of a chatbot used by a university institution administration office to answer frequently asked questions from students to reduce the strain on administration officers, as well as make it easier on the students. The researcher observed that students would normally be inconvenienced by having to spend money to get to campus, and then wait in lines just to obtain information. The bot developed in this research aimed to mitigate these issues, especially in periods of high concentration of students with queries, such as during the registration periods.

In support of this study, the proposed chatbot system adopts similar NLP APIs to process the user input and was deployed on the Facebook Messenger platform. Several features and developmental processes were adopted from this research, including the use of JavaScript to code the bot, and JSON files to store the intents set by the developer. However, the researcher made use of Dialogflow as the primary NLP API, while the implemented system made use of Wit.ai. This is because Wit.ai was acquired by Facebook, and as such made it simpler to integrate with the code. Additionally, the researcher adopted an agile software development methodology, which was found to be a suitable development methodology for this type of system. The paper demonstrated the validity of such systems on the Facebook messenger platform, which expands the horizon of the user to anyone with a Facebook account and internet access.

Similar to the Interactive Text-Based Query Chatbot System described above, the research done by [13] also describes the development and design of a chatbot used to answer frequently asked questions from students of the University of Namibia. This paper however provides an alternative means of implementation, making use of a custom-built site, using XAMPP as opposed to integrating with Facebook messenger. The development methodology applied also differs in that this paper uses Rapid Evolutionary Prototyping, which is similar to agile methods, however, focuses on releasing prototypes quickly and iterating them until the final design is complete.

For the system to process and generate responses, the researcher made use of a dataset-builder algorithm and a keyword matching algorithm. The process of the dataset-builder involved manually inserting a frequently asked question twice with variations in phrasing, as well as the answer. Then, the algorithm splits each question into separate words, extracted keywords from the query, and generates a data set by "saving the differently phrased questions, extracted keywords and the corresponding answer as a record into MySQL". Following this, when running the chatbot, the keyword matching algorithm would take in a query from a user, get the stored dataset from MySQL and compare each word of the user's query to the keywords from the dataset. Once a match was found, it would take the stored response and display it to the user.

However, the paper reports that this algorithm failed to process complex questions. In contrast, the implemented counseling chatbot made use of more powerful pattern-matching algorithms 
found in the Wit.ai API. This algorithm involved manually setting intents of a few user inputs, and then coding the required responses. The API was then able to automatically identify intents from user queries with minimal supervision from the developer and was able to understand intents even with varied phrasing in the query. Despite these gaps, this paper provided a source of information on types of chatbots and their characteristics, which was beneficial when beginning research in the domain.

In the paper, [14] design and develop a chat application that allows students, faculty members and other users to interact with an AI system that provides information based on college related activities. The bot is implemented as an Android application and features a chat interface, in which the user can query the bot for information, and a notifications interface, in which the latest events are displayed.

Similar to this paper, the implemented career counseling system is also implemented as a text-based interface and makes use of a similar NLP algorithm. The NLP tool used in their research extracts redundant words from the user input and identifies intents and entities within the remaining words of the query. The intents are compared with data stored in an admin-defined dataset, and an appropriate response is selected. According to [14], the process is shown in the Figure 2.

This flow diagram was adopted into the research. However, in this research the responses are stored in a separate database, while the dataset implemented in the career counseling system is stored within the NLP tool system implemented, i.e. Wit.ai, itself. Additionally, while their NLP tool removes redundant words from the queries, Wit.ai functionality can automatically identify relevant words from a query without needing to remove the rest. When compared to the flowchart or working flow presented in Figure 2, this process flow was found to fit the requirements of the career system implemented and was as such adopted into the research.

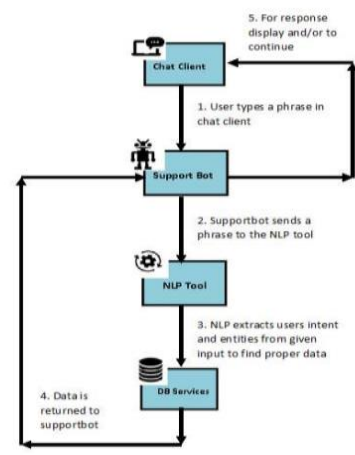

Figure 2: The Working flow of SupportBot

\section{Methodology}

In this section, the research methods and approached applied in this study are discussed in section 5.1 and 5.2.

\subsection{Research Methodology}

This research adopted a co-design approach of research methodology and a software development methodology. According to [15], a developmental research methodology is defined as "the systematic study of designing, developing and evaluating instructional programs, processes and products that must meet the criteria of internal consistency and effectiveness". The research was aimed at establishing new procedures and tools based on methodical analysis of specific cases. The software was developed using an agile software development methodology. This is defined by [16] as an incremental development method in which increments are "small and, typically, new releases of the system are created and made available... every two to three weeks". This was selected as its flexibility and iterative development process was most suitable for this type of work. Additionally, it allows for changing the scope of requirements during the development process [17].

5. 1.1. Data Collection. The study made use of both qualitative and quantitative data. A survey was conducted at the onset of development to gather information about individuals' opinions regarding their possible career choices, and what types of career advice they would like to receive from the system. This data was used to prove hypotheses postulated earlier on in the paper regarding any correlation between career guidance and career satisfaction as well as assisting in the development of the features of the chatbot. The data was also used to determine the structuring of the questions asked by the bot.

5.1.2. Sampling Frame. A sampling frame is defined by [18] as a list of the whole population of participants that could be included in the survey, from which a sample will be chosen. In this research, the sampling frame was senior university students and individuals who had recently left university. The survey made use of a convenience sampling method, in which the survey was shared in university social media groups, and to those the researcher knew had recently graduated. Those who were willing and able to respond then filled out the survey and submitted their results. Finally, a sample of 36 participants was compiled.

5.1.3. Survey Results. This section evaluates the results of the survey carried out on the 36 participants of the sample. The majority of respondents ranked a 3 when asked how clear they were about their career goals, where a 1 was "very clear" and a 5 was "very unclear". Similarly, the graph depicted in Figure 3 shows skews to the right, with $36.1 \%$ of participants selecting either a 4 or 5 , and $30.6 \%$ choosing a 1 or a 2 . This would suggest that career guidance would be beneficial to help users gain clarity on their career goals, and on what their jobs would entail.

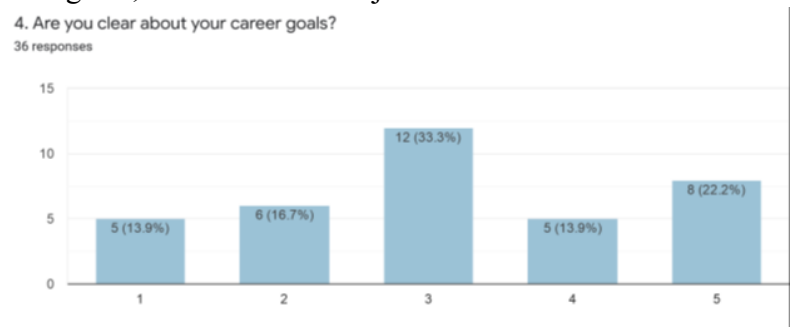

Figure 3: Clarity about the career goals 
Figure 4 depicts the type of service that the receiver wants from the career counsellor. With $83.3 \%$ of votes, the most popular choice for the type of advice the respondents would like to receive was career descriptions. Following this, $75 \%$ found that resources on how to prepare for interviews were vital. These results were taken into consideration when determining what features to incorporate into the chatbot.

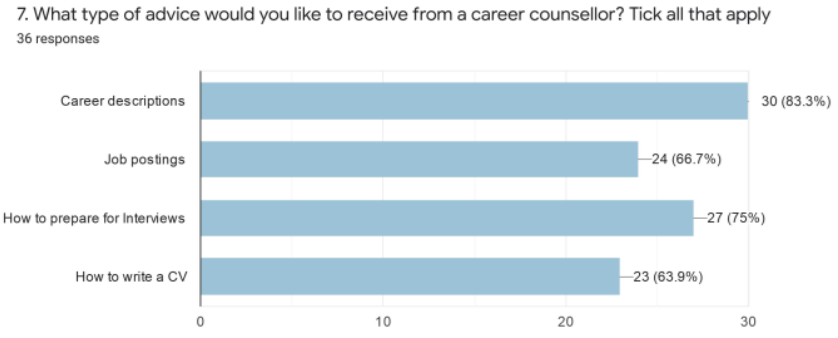

Figure 4: type of service that the receiver wants from the career counsellor

\subsection{Developmental Methodology}

The chatbot system was developed on the Facebook Messenger platform, using tools such as the Facebook SDK, the Messenger Platform APIs, JSON files and JavaScript, and AI using Natural Language Processing.

The system was built using AI algorithms that analysed user responses and provided a suitable response. The application utilized the Facebook Messenger platform and AI tools found on the platform Wit.ai to perform this function. The user was able to request different resources from the chatbot, such as $\mathrm{CV}$ templates, aptitude tests, and so on, and the chatbot was able to apply NLP to understand the messages and return an appropriate response. The system architecture is shown in Figure 5.

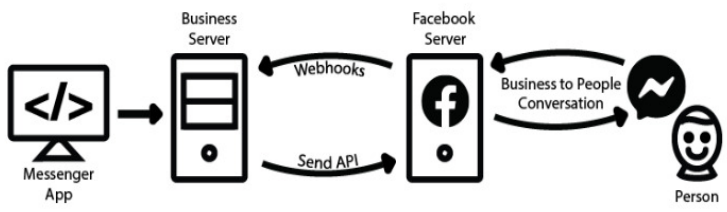

Figure 5: System Architecture

\subsubsection{Design Phase}

The system was developed using an agile development process. Data collected from the sample in the research methodology stage was used in requirements gathering, as the criteria for an iteration of software development, referred to herein as "user stories". The milestone for the first phase is to select which user stories served as the basis of development for the given iteration, or "sprint".

In the second phase, the user stories were broken down into tasks. The goal of this phase was to analyse the user stories and translate them into a quantifiable list of tasks to be implemented. The next three phases of planning, developing, and releasing the software involved implementing the tasks quantified in phase two. The milestone of this was the given iteration's release of the software. The next phase involved evaluating the system to determine whether the features perform as intended and get user feedback.
The milestone of this phase was the user feedback. As it was not feasible to get feedback from each participant that took part in the survey, a small number of participants were selected as testers of the system during the development process.

After the iteration was released and evaluated, another user story was selected to drive the next sprint, and so on until the final version was completed.

\subsubsection{Implementation phase}

In the implementation phase, the tasks created in the design phase were used as specifications for development. With each iteration of development, actionable tasks were outlined and carried out. Once coding was completed, they were tested to make sure they sufficiently achieved the goal of the user story before development entered the next iteration cycle. This process was repeated until all the features had been implemented, and the system was seen to satisfy the research objectives.

The tools required to implement the chatbot are listed as follows:

1. Node.js. This was used in the chatbot development.

2. Glitch. An IDE is used as the development platform and to host the server of the application.

3. JSON files. These were used as a data store for the chatbot responses

4. Wit.ai. An AI tool that implemented NLP in the chatbot so that the bot could understand user input.

5. Express. Used to create the server, and acts as the API

6. Facebook Messenger. This was the main user interface through which the user could interact with the chatbot

After the webhook was set-up, it was linked with the Facebook page. To do this, it was required to create a Facebook page from which the chatbot would run and set up a Facebook developer account. This gave access to the tools that were used to set up and run a chatbot on the Messenger platform. A Facebook app was created with the purpose of creating a page and managing various business integrations, as shown in Figure 6.

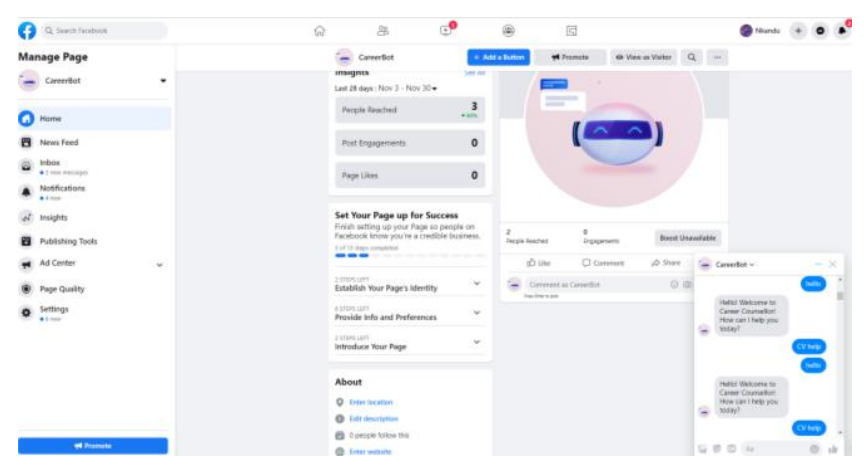

Figure 6: CareerBot Facebook Page

\subsubsection{Testing phase}

At each increment of design, the features being implemented were tested during the coding phase, as well as once the increment was completed. Figure 7 below shows an early iteration of the system, in which it was tested to ensure that it could receive messages and send a response. 


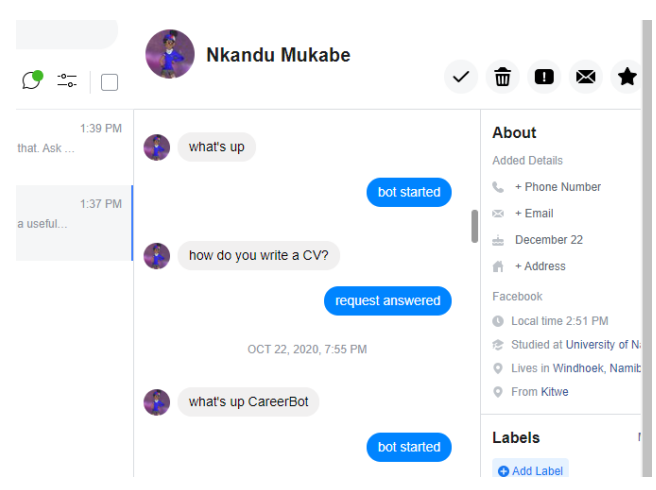

Figure 7: Testing phase to determine responses

\section{Results}

This section gives a brief overview of the results of the study, and the product of the software development. The development was completed and the chatbot system was shown to be operational. It is accessible via Facebook messenger, allowing anyone with an account and internet access to use its services. It currently can respond to user input and provide resources such as CV templates, resources on preparing for interviews, job listings and an aptitude test. Future functionality of the features and the operational screenshots shown in Figures 8 to 13 below present the chatbot in use and how it responds to user messages.

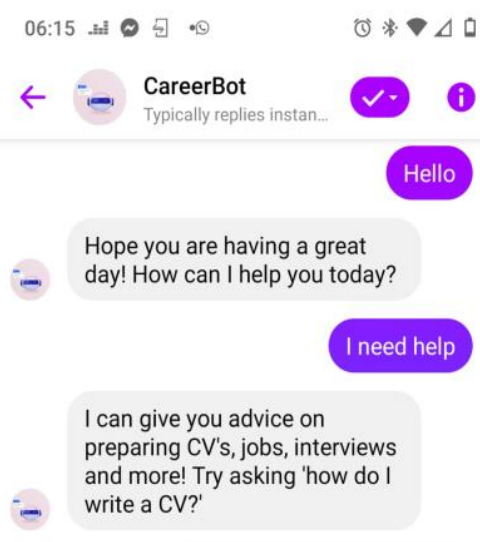

Figure 8: Initiating conversation with the Chatbot

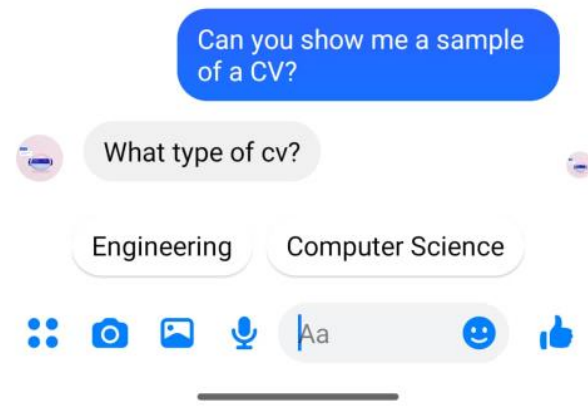

Figure 9: The bot returning CV resources based on field of study

\section{How do I write a cover letter}

I think this might be helpful!

(1) $\mathrm{b} 3 \mathrm{f} 77468-\mathrm{b} 79 \mathrm{a}-4 \mathrm{c} 54-8 \mathrm{e} 1 \mathrm{c}-514$

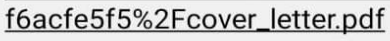

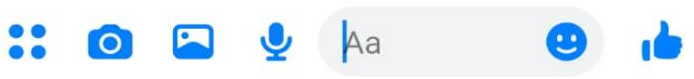

Figure 10: The bot returning resources for cover letters

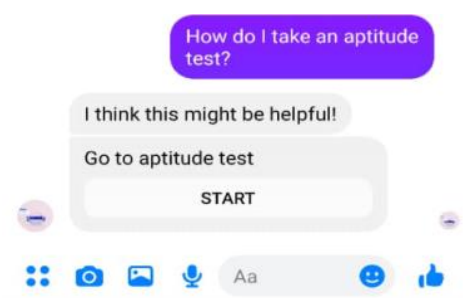

Figure 11: The bot returning a web-view for an aptitude test

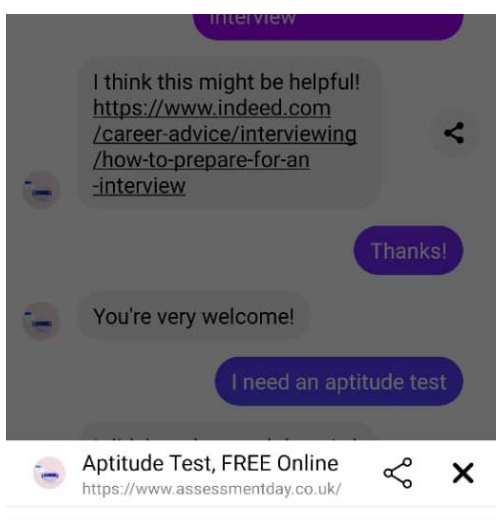

\section{Aptitude Test Practice}

Practise the tests employers use

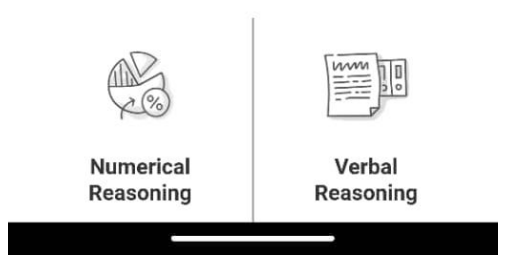

Figure 12: The bot returning a web-view for an aptitude test 
I didn't understand that. Ask me something like 'how do I write a CV?'

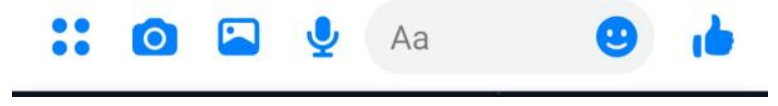

Figure 13: Exception is thrown when the bot does not understand a message

In summary, the results obtained were found to adequately meet the requirements of the system, and the system testers were satisfied with the responses received. The bot showed a fast response time, returning results in a few seconds. However, it was noted that the response time was considerably slowed based on the user's internet connection, and an unstable internet connection could slow the responses from nearly real-time, to several seconds. Additionally, as seen in Figure 13, the NLP demonstrated difficulty in processing certain input phrases such as single word messages, or messages outside the scope of its programming. In these cases, an exception was thrown, and the bot suggested a phrase to guide the user back to topics within its scope.

\section{Discussions}

The application developed can provide a variety of career counseling services to the users, including assistance with writing CVs and cover letters, and preparing for interviews. In addition, it has the functionality to find job listings based near the user and provide an aptitude test that allows the user to identify which career would be best suited for them.

In accordance with the second objective, the appropriate tools were chosen to facilitate the operation of the chatbot system.

1. The API used for NLP is called Wit.ai, which is "a natural language interface for applications capable of turning sentences into structured data" [19]. This was used to process user queries and provide appropriate responses

2. Additionally, the system made use of the Facebook messenger API to enable the sending of messages by the user and allowing the bot to respond

3. The system was coded in JavaScript and made use of the Facebook Developer SDK.

4.JSON files were used as a data store to store the responses sent by the chatbot.

A peer-to-peer system was developed that could understand user input and respond with the appropriate resource that the user asked. This was done with the use of the NLP functionality of the Wit.ai API. It used built-in pattern-matching algorithms to identify an intent within a message sent by and determine what the user was requesting. Then the bot would provide the appropriate resource based on the identified intent.

\section{Conclusions and Recommendations}

In conclusion, this research was aimed at the development of a chatbot that would give career counseling advice to senior university students and graduates. This was the main research objective, which was achieved by the designed system. A survey was carried out on students and graduates that was used to identify which features to incorporate into the chatbot. At the end of the development phase, a few participants of the survey were selected to test the bot to determine if the system produced a satisfactory output. Additionally, the objective of designing an automated system that takes in queries from the user and provides them with appropriate resources as output was also achieved. A variety of tools were used to achieve this, including the Facebook Messenger platform and Natural Language Processing. The result is a chatbot that responds very quickly to user messages and provides useful resources based on their queries.

In addition, the bot was able to understand the intent of the user sent messages and provide a suitable response. With the use of this system, users can be better informed in navigating activities involved in finding careers that they may not otherwise understand. Overall, the bot shows a lot of potential to be integrated into existing institutions such as universities and other higher learning institutions to provide access to a service that many people have gone without for too long.

Below are the few further developments that could be incorporated to the chatbot system to add to the robustness of the system. This list is not exhaustive, and many other features could be applied as well.

1. Voice-to-text functionality

Similar to the systems designed by [5], [8], [10] and more, the system could allow for users to use speech to interact with the bot. The user could use either voice or text input when talking to the bot, and in turn, the chatbot could respond with either text or voice. 2. Integrate a system for filtering abuse/bad language

The chatbot system could be integrated with a system that filters out inappropriate or abusive language sent in by users. This is to maintain appropriate interactions with the bot and ensure that the AI does not inadvertently train itself on inappropriate language.

3 Integrate it to a website

Currently, the bot operates on a standalone Facebook page, however, in the future it can be integrated on an organization's website such as on a university's website or a dedicated counseling website for further ease of access for the users.

4. Integrate counseling personnel as live agents

A feature can be added to the bot to allow the user to connect to a real counsellor for help with complex queries and concerns that may fall outside the capabilities of a chatbot.

5. Enable the use of the bot in different languages

The bot can be further designed to interact with users in languages other than English so it could be integrated even in non-Englishspeaking institutions or organizations.

\section{Limitations of the study}

A few limitations were identified in the process of developing the system and are outlined below:

1. The chatbot required a good internet connection for very fast responses. When the tester had a good internet connection, the chatbot was observed to respond very quickly, almost in real 
- time. However, if the tester's internet connection was unstable, the chatbot showed a notable delay of 5-10 seconds in responding.

2. The chatbot was only able to interact in English. Due to time constraints, it was impossible to include functionality for the user to interact with the chatbot in a language other than English

3. The scope of the chatbot's functionality had to be limited to a few resources in select fields due to the vast majority of possible job types. It was impossible to model responses for every single career field and every single resource that could feasibly be asked for by a user.

4. This study could not evaluate how well the system could hold up against a large number of concurrent users at one time

5. The system was also limited to Facebook users only rather than open to anyone, and as such one could not use the system if they did not have a Facebook account

\section{ACKNOWLEDGMENTS}

Thanks are also reserved to the institutions, School of Computing University of Namibia (UNAM) for making resources available for conducting this study.

\section{REFERENCES}

[1] Omarjee, L. (2019). SA professionals unhappy with their jobs - survey. Retrieved May 3, 2020, from Fin24: https://www.fin24.com/Economy/sa-professionalsunhappy-with-their-jobs-survey-20190608

[2] Copeland, B.J. (2017, Jan). Artificial Intelligence. Retrieved from Britannica: https://www.britannica.com/technology/artificial-intelligence

[3] Chatbot: What is a Chatbot? Why are Chatbots Important? (2018). Retrieved from Expert System: https://expertsystem.com/chatbot/

[4] Chowdhury, G. G. (2005). Natural Language Processing. Annual Review of Information Science and Technology, 37(1), 51-89. doi:https://doi.org/10.1002/aris.1440370103

[5] Ohm, A., \& Bhavani, K. (2019, June). Chatbot for Career Guidance Using AI International Journal of Computer Sciences and Engineering, 7(6), 856-860. doi:https://doi.org/10.26438/ijcse/v7i6.856860

[6] Mamgain, D. (2019, July 12). Dialogflow vs. Lex vs. Watson vs. Wit vs. Azure Bot. Retrieved October 31, 2020, from DZone: https://dzone.com/articles/dialogflow-vs-lex-vs-watson-vs-wit-vs-azure-bot-wh

[7] Vichare, A., Gyani, A., Shrikhande, Y., \& Rathod, N. (2015, October). A chatbot system demonstrating Intelligent. International Journal of Advanced Research in Computer Engineering \& Technology (IJARCET), 4(10), 3783-3785.

[8] Parab, A., Palkar, S., Maurya, S., \& Balpande, S. (2017, March). An Intelligent Career Counselling Bot: A System for counselling. International Research Journal of Engineering and Technology (IRJET), 4(3), 2325-2330.

[9] Bhartiya, N., Jangid, N., Jannu, S., \& Shukla, P. (2019). Artificial Neural Network Based University Chatbot System. IEEE Bombay Section Signature Conference (IBSSC), 1-6. doi:978-1-5386-7401-7/19

[10] Banerjee, A. (2019, January). Text and Voice-Enabled Chatbot Enhancing the User Experience in Career Counselling Domain. Dublin, Ireland.

[11] Chopde, K., Mondhe, S., Sahu, N., \& Deshbhratar, S. (2017). PCE College Enquiry Bot. International Journal of Innovations in Engineering and Science, 2(1), 1-4.

[12] Egumbo, T. (2019, April). Interactive Text Based Query Chatbot System: To Help Answer Student Frequently Asked Questions At the University of Namibia. Windhoek, Namibia.

[13] Goeiemann, W. (2019, November). UNAM Assistant: A Rule-Based Chatbot Windhoek, Namibia.

[14] Niranjani, V., Nivethitha, K., Preetha, S., \& Sangeetha, S. (2018). SupportBot: An Artificial Agent Useful for Gathering College Details. International Journal of Innovative Research in Computer, 6(1), 413-418. doi: 10.15680/IJIRCCE.2018. 0601069

[15] Richey, R. C., \& Klein, J. D. (2005). Developmental Research Methods: Creating Knowledge from Instructional Design and Development Practice. Journal of Computing in Higher Education, 16(2), 23-38. doi:10.1007/BF02961473

[16] Sommerville, I. (2016). Software Engineering (10th ed.). Harlow, United Kingdom: Pearson.

[17] Waterfall Vs. Agile: Must Know Differences. (2020). Retrieved from Guru99 https://www.guru99.com/waterfall-vs-agile.html
[18] Oates, B. J. (2006). Researching Information Systems and Computing. Sage Publications Ltd. doi:978-1-4129-0223-6

[19] Giulia. (2017, July 2). Wit.ai and how to use it. Retrieved October 24, 2020, from Medium: https://medium.com/@Giuul/wit-ai-and-how-to-use-it-72372b07d98b 